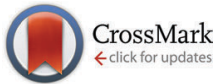

Cite this: New J. Chem., 2016, 40,7210

Received (in Montpellier, France) 13th February 2016, Accepted 17th June 2016

DOI: 10.1039/c6nj00353b

www.rsc.org/njc

\title{
Silanated nano ZnO hybrid embedded PMMA polymer coatings on cotton fabrics for near-IR reflective, antifungal cool-textiles $\dagger$
}

\author{
S. Soumya, ${ }^{a}$ S. Nishanth Kumar, ${ }^{b}$ A. Peer Mohamed $^{a}$ and S. Ananthakumar*a
}

\begin{abstract}
Textile surfaces engineered with infrared/ultraviolet energy shielding coatings are an emerging technology in the processing of solar heat protective cool-textiles. In this work, such multifunctional coatings were prepared using silane treated nano ZnO hybrid embedded PMMA colloids on a model black colored cotton textile. Black cotton fabrics were selected for the study because they exhibit a high heat-build up tendency due to the inherent black body radiation effect. 3-(Aminopropyl)trimethoxy silane modified nano $\mathrm{ZnO}$ hybrids (APZO NHs) were first prepared and transformed into a stable colloidal dispersion in a PMMA medium to obtain an APZO NHs/PMMA sol. This hybrid-polymer colloidal sol was dip-coated on the fabric surface to form multilayer coatings. This surface engineered black cotton was subjected to studies of its phase analysis, chemical interaction, and morphological features in addition to its NIR reflectance, UV shielding efficiency and antifungal properties. The NIR/UV reflectance performance was analyzed with respect to the number of coatings and compared with the standard white cotton fabric which has roughly $50 \%$ NIR reflectance. When silane treated ZnO hybrid particulates are embedded in the PMMA matrix, the polymer coatings offer mechanically stable, UV/NIR radiation shielding textile surfaces in the wavelength region between 360 to $1600 \mathrm{~nm}$ that eventually turns the black cotton to cool-black textiles. The coatings also offer hydrophobic functionality as well as strong protection against the growth of Aspergillus flavus and Aspergillus niger fungi species resulting in biosafe textiles.
\end{abstract}

\section{Introduction}

Textiles engineered with functional surface coatings using inorganic as well as metallized nanostructures successfully offer shielding effects for electromagnetic and infrared radiation energy. These kinds of engineered textiles find applications in the military as well as in strategic fields. ${ }^{1-5}$ In continuation of this research, attempts are being made to develop functional textiles that possess UV protection capability, sunlight active photo catalytic selfcleaning properties, and solar heat absorption as well as solar heat reflection qualities. ${ }^{6-12}$ Recently, textile industries have also shown great interest in surface engineering of textile products that can efficiently repel oily substances, organic dirt and even mosquitoes. This research is further extended to develop textiles with

\footnotetext{
${ }^{a}$ Functional Materials Section, Materials Science and Technology Division, Council of Scientific and Industrial Research-National Institute for Interdisciplinary Science and Technology (CSIR-NIIST), Thiruvananthapuram-695019, Kerala, India. E-mail: ananthakumar70@gmail.com; Fax: 91-471-2491712; Tel: 91-471-2515289, 91-9497271547

${ }^{b}$ Agro Processing and Natural Products Division, Council of Scientific and Industrial Research-National Institute for Interdisciplinary Science and Technology (CSIR-NIIST), Thiruvananthapuram-695019, Kerala, India

$\dagger$ Electronic supplementary information (ESI) available. See DOI: 10.1039/c6nj00353b
}

bio-mimetic camouflage coatings; as well as smart coatings with water and detergent free cleaning technology. ${ }^{1,2,13-15}$

Near-IR reflective coatings in order to minimize solar heat penetration are an important requirement for human beings. Textiles with such coatings can protect a large number of casual workers who engage in building construction and are exposed to sunlight in countries like India and the Gulf regions. Surface functional coatings processed using inorganic metal oxides such as $\mathrm{TiO}_{2}, \mathrm{ZnO}$, and $\mathrm{CeO}_{2}$, which are well recognized white pigments, have been largely studied on ceramic tiles and metal roofing sheets to prove their cool-functional properties. ${ }^{16-20}$ Recently, they also have been tested on textile and leather surfaces to incorporate antibacterial/antifungal, and anti-wrinkle properties. ${ }^{13,21,22}$ Since normal white pigments reflect $>80 \%$ of the incident sunlight radiation they are recognized as ideal activepigment components for developing cool-coatings on textiles.

$\mathrm{ZnO}$ is a known pigment, that has been extensively patented for cool-paint products for building materials, mainly the metal/ polymer roofing surfaces. ${ }^{23-26} \mathrm{ZnO}$ is a non-corrosive material, non-toxic via ingestion and inhalation, that is also known for its UV absorption, hydrophobic qualities and high near-infrared reflectivity. ${ }^{5,27-33}$ Chemically cross-linked nanohybrids offering near-infrared radiation reflectance in the critical wavelength 
region $700-2500 \mathrm{~nm}$ in the solar spectrum are the best way to apply cool-coatings on textiles. In situ growth of nano $\mathrm{ZnO}$ on the cotton fabric is a previously reported strategy. ${ }^{22}$ However, poor mechanical adhesion is realized in such coatings. One of the most interesting ways to overcome this issue is perhaps the concept of using functional hybrid coatings processed from chemically capped hybrid nanodispersoids embedded within any polymer matrix. Organic capping molecules can chemically react with cellulosic units and promote better chemical bonding with textile surfaces. Poly(methyl methacrylate) [PMMA] is a bio-inert polymer with low thermal conductivity $\left(\lambda=0.2 \mathrm{~W} \mathrm{~m}^{-1} \mathrm{~K}^{-1}\right)$ and high optical transparency throughout the visible range in addition to high mechanical stability. Bulk PMMA/ZnO nanocomposites have previously been explored to understand their beneficial properties like UV blocking, optical transparency, tunable refractive index, wear resistance, and thermal stability. ${ }^{31,32,34-37}$ The results strongly indicate PMMA polymer is a probable matrix for fabricating UV/NIR shielding transparent coatings. There are no reports on the application of silane modified ZnO nanohybrids/PMMA coatings on textiles that provide a NIR control cooling effect.

In this work, assembly of silane treated nano $\mathrm{ZnO}$ hybrid encapsulated PMMA coatings onto the surface of the black cotton fabrics has been attempted to impart a NIR reflectance quality. A colloidal sol prepared from silane treated $\mathrm{ZnO}$ hybrid particulates in a PMMA medium created nanometer thin multilayer coatings on the non-planar textile fabric surfaces via physico-chemical and electrostatic interactions. In case of coatings designed with such silane/ZnO/polymer multiphase architectures, ample opportunity exists to incorporate any active functional molecules like proteins. Recently reported research articles clearly showed multilayered/ multiphase topcoats can produce surface properties including UV protection, durability, hydrophobicity/hydrophilicity and antimicrobial activity. ${ }^{38-41}$ Here, we demonstrate NIR reflective silane ZnO coatings on textiles for developing cool-clothes. The NIR shielding properties of silane treated nano ZnO embedded PMMA polymer coatings on black cotton fabrics were systematically evaluated and reported. In situ silane cross-linked nano $\mathrm{ZnO}$ hybrid particles were first synthesized via chemical precipitation. The mol\% of silane was varied as $0,1,2,5$, and $20 \%$. A stable silane modified $\mathrm{ZnO} / \mathrm{PMMA}$ colloid was then prepared and thin films of hybrid coatings on cotton fabrics were developed by simple dip-coating, via layer-by-layer (LbL) technique. The effect of silane on the $\mathrm{ZnO}$ nanoparticle size, surface charge characteristics, crystallinity and chemical bonding were studied. Bulk NIR reflectance and total solar reflectance $\left(\mathrm{TSR}=R^{*}\right)$ in the wavelength range of 700-1600 $\mathrm{nm}$ were evaluated. The results are correlated with silane concentrations and coating layers. The coated fabric was also briefly examined for antifungal properties.

\section{Experimental}

\subsection{Materials and reagents}

Black as well as white colored cotton cloth pieces with a size of $6 \mathrm{~cm} \times 6 \mathrm{~cm}$ were cleaned first ultrasonically and then with ethanol and distilled water before use. Zinc acetate dihydrate
$\left(\mathrm{Zn}(\mathrm{OAc})_{2} \cdot 2 \mathrm{H}_{2} \mathrm{O}\right.$; Merck Specialities Pvt. Ltd, India, purity $\geq 98 \%$ ), sodium hydroxide (NaOH; Merck, India, 99\%), (3-aminopropyl)trimethoxysilane (APS; Sigma-Aldrich, India, purity $>99 \%$ ), and poly(methyl methacrylate) (PMMA; Sigma-Aldrich) with an average $M_{\mathrm{w}} 996000$ were used as supplied. The fungal pathogens used for the antifungal study were Aspergillus niger MTCC 2756 and A. flavus MTCC 183. The fungal strains were procured from Microbial Type Culture Collection (MTCC) and Gene Bank, Institute of Microbial Technology, Chandigarh, India. The fungal strains were maintained on PDA agar slants at $4{ }^{\circ} \mathrm{C}$. Toluene, ethanol, and acetone were selected as solvents. Double distilled water was utilized for all the preparation.

\subsection{Synthesis of (3-aminopropyl)trimethoxysilane modified nano ZnO hybrids (APZO NHs)}

Silane modified ZnO colloidal particulates were synthesized by a co-precipitation technique as reported earlier. ${ }^{42} 0.1 \mathrm{M}$ zinc acetate dihydrate $\left[\mathrm{Zn}(\mathrm{OAc})_{2} \cdot 2 \mathrm{H}_{2} \mathrm{O}\right]$ aqueous homogeneous solution was first prepared. To this solution, different amounts of 3-aminopropyl-trimethoxy silane were added in order to reach $\mathrm{Si} / \mathrm{Zn}$ molar ratios of $0,1,2,5$, and $20 \%$, respectively. The solution of $1 \mathrm{M} \mathrm{NaOH}$, prepared in distilled water at room temperature, was added dropwise with constant stirring to maintain a $\mathrm{pH}$ of 10. At this $\mathrm{pH}$ condition, a white precipitated gel was obtained and stirred mechanically for $4 \mathrm{~h}$. Later, the gel mass was centrifugally washed using distilled water at $3000 \mathrm{rpm}$ and then washed once with ethanol in order to remove any excess organosilane and a large part of un-reacted residual acetates. The gel mass was finally dried at $60{ }^{\circ} \mathrm{C}$ overnight in an electrically heated oven. The obtained silanized $\mathrm{ZnO}$ nanohybrids were designated as $x \%$ APZO NHs, where $x$ indicates the $\mathrm{Si} / \mathrm{Zn}$ molar ratio used. The untreated phase pure ZnO nanoparticle (0\% APZO NHs) was also prepared for studying the comparative performance and the effect of silane.

\subsection{Fabrication of APZO NHs/PMMA nanocoatings on cotton fabrics}

APZO NHs/PMMA coating solution was prepared by dispersing $0.1 \mathrm{wt} \%$ silane modified $\mathrm{ZnO}$ nanohybrids in $60 \mathrm{~mL}$ toluene. Similarly, $10 \mathrm{wt} \%$ PMMA was also dissolved separately in a toluene medium. Both solutions were mixed, sonicated for $30 \mathrm{~min}$, and further stirred magnetically overnight in order to ensure homogeneous mixing. A stable silane modified ZnO/PMMA colloidal sol was successfully obtained and further applied to the white and black colored cotton fabrics via dipping-withdrawing coating technique. The cotton fabric pieces of $6 \times 6 \mathrm{~cm}$ were immersed in the coating solution. The fabric was soaked in the coating precursor sol for $2 \mathrm{~min}$ in order to ensure the better uptake of nano ZnO layers. After coating, the cloth was dried by hanging at room temperature and cured to obtain APZO NHs/PMMA functional coatings. The dip-coating treatments were repeated 1 to 7 times and finally 20 times to obtain 20 layer coatings. Simultaneously, neat PMMA coatings and ZnO/PMMA coatings without any silane were also made for reference. For convenience, specific codes were given to the coated cotton fabric samples as follows: bare white cotton (bare WC), bare black cotton (bare BC) 


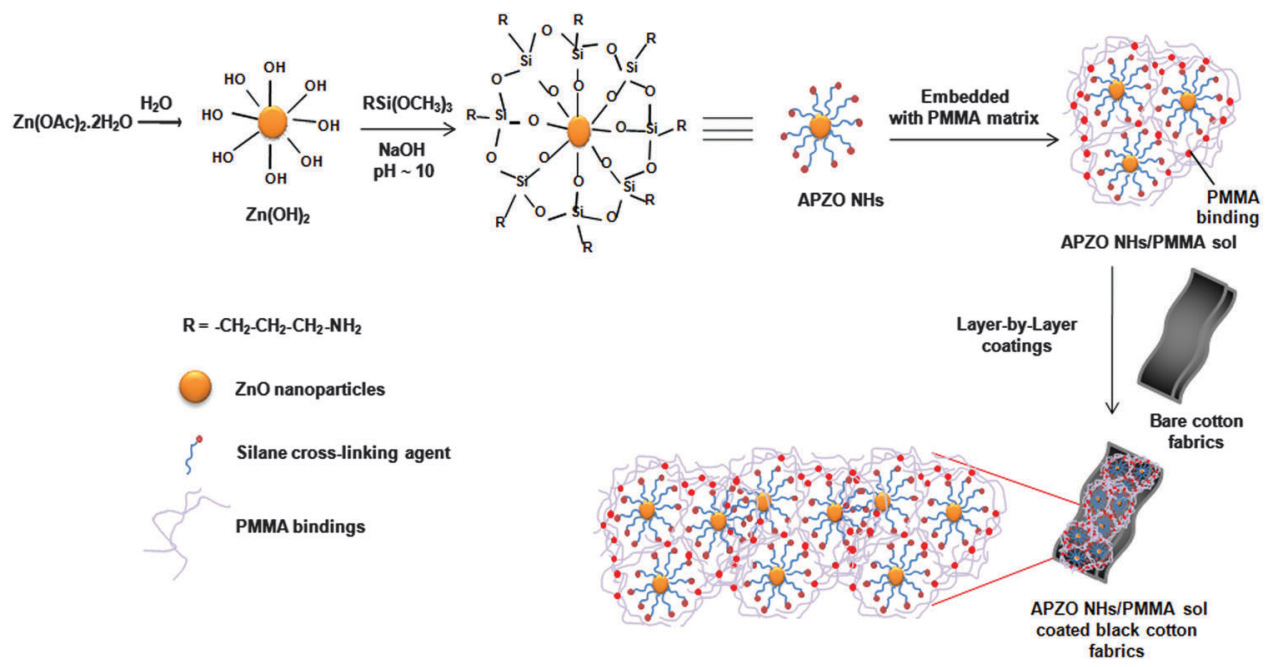

Fig. 1 Procedure and chemical interaction involved between the reactants; silane capping molecule, nano ZnO and PMMA polymer matrix on the cotton fabrics.

and after neat PMMA coating treatments, PMMA/WC, PMMA/BC; the same with silane treated nano ZnO: APZO NHs/PMMA/WC and APZO NHs/PMMA/BC. The samples with varying mol\% APS concentration were represented as $0,1,2,5$, and $20 \%$ APZO NH. Fig. 1 schematically described the chemical interaction involved between the reactants: the silane molecule, nano ZnO and PMMA polymer matrix on the cotton fabrics.

\subsection{Characterisation}

The crystalline nature and phase purity of the nano APZO NHs were characterized by powder X-ray diffraction using an X'Pert Pro, Philips X-ray diffractometer equipped with $\mathrm{Cu} \mathrm{K}_{\alpha}$ radiation $(\lambda=1.5406 \AA)$ in the $2 \theta$ range 10 to $70^{\circ}$ with a 0.02 scan increment. Particle size distribution and zeta potential were measured by dynamic light scattering (DLS) using a Malvern Zetasizer Nano ZS (Worcestershire, UK). For these measurements, APZO NH particulate colloids were prepared in aqueous media by dispersing $0.01 \mathrm{wt} \%$ APZO NHs and subsequently de-agglomerated through ultrasonication for $15 \mathrm{~min}$ in an ultrasonicator (Ultrasonic Processor P2, Vibronics Pvt. Ltd, Bombay, India). A Mettler Toledo pH meter (Model $\mu$-8603) was used for the $\mathrm{pH}$ measurements. Thermogravimetric analysis (TGA) of the APZO NHs was conducted using a TG 50 Shimadzu TGA in air.

The chemical interaction of silane with nano $\mathrm{ZnO}$ hybrid particulates was performed using an IR Prestige-21, Shimadzu FTIR spectrophotometer in the scanning range of $4000-400 \mathrm{~cm}^{-1}$ using a standard $\mathrm{KBr}$ pellet as reference. In APZO NHs/PMMA treated cotton fabrics, the chemical interaction was recorded on a Bruker alpha ATR-FTIR spectrometer instrument using attenuated total reflectance (ATR) mode with a resolution of $4 \mathrm{~cm}^{-1}$ accumulating 24 scans. The microstructure and morphological features of APZO NHs and APZO NHs/PMMA treated cotton fabrics were examined using a ZEISS EVO 18 Special Edition scanning electron microscope (SEM) operated at $20 \mathrm{kV}$. The chemical composition was ascertained using energy-dispersive X-ray (EDX) mapping.
The transmission electron microscopic (TEM) image analysis and selected area electron diffraction (SAED) of the APZO NHs were performed on a FEI Tecnai 30G2S-TWIN transmission electron microscope operated at an accelerating voltage of $300 \mathrm{kV}$. The surface roughness behavior of bare and APZO NHs/PMMA coated cotton fabrics was obtained by atomic force microscopy (AFM) analysis (Bruker Multimode, Germany) in the tapping mode and was performed at a scan size of $20 \mu \mathrm{m} \times 20 \mu \mathrm{m}$.

\subsection{NIR/UV shielding analysis}

The optical properties of the APZO NHs and APZO NHs/PMMA/ cotton fabrics were monitored using a Shimadzu UV/vis spectrophotometer (UV 240 IPC) in the wavelength range of 200 to $800 \mathrm{~nm}$. The absorption coefficient $(\alpha)$ is evaluated using the equation $\alpha=A / d_{\mathrm{s}}$, where $A$ is the measured absorbance and $d_{\mathrm{s}}$ is the thickness of the sample in the UV-vis cell $(0.4 \mathrm{~cm})$. The absorption coefficient is related to the energy band gap $\left(E_{\mathrm{g}}\right)$ by: $(\alpha h \nu)^{2}=E_{\mathrm{D}}\left(h \nu-E_{\mathrm{g}}\right)$, where $h$ is Planck's constant, $\nu$ is the frequency of the incident photon, $E_{\mathrm{D}}$ is a constant and $E_{\mathrm{g}}$ is the direct band gap. If $(\alpha h \nu)^{2}$ is plotted versus $h \nu$ over the absorption wavelength range, the energy band gap $\left(E_{\mathrm{g}}\right)$ can be calculated by extrapolating the linear region of the curve to $\alpha=0$. The band gap values were calculated from the absorbance spectra. The value of wavelength, $\lambda$ (in $\mathrm{nm}$ ), corresponding to the absorption edge is substituted into the formula, $E_{\mathrm{g}}$ (in $\mathrm{eV}$ ) $=1239.8 / \lambda^{31,32,43}$ The photoluminescence spectra of the APZO NHs dispersed in a water medium were acquired using a Cary Eclipse fluorescence spectrophotometer (Varian India Pvt. Ltd) equipped with a liquid sample holder accessory at an excitation wavelength of $330 \mathrm{~nm}$.

The near-infrared diffuse reflectance (700-2500 nm) spectra of the bulk APZO NHs and APZO NHs/PMMA coated fabrics were analyzed using a Shimadzu UV-vis-NIR spectrophotometer (UV-3600) with an integrating sphere attachment (ISR-3100). The instrument was calibrated using the reference poly(tetraflouroethylene) (PTFE). As reported in the literature, ${ }^{31,32,44,45}$ the NIR solar reflectance $\left(R^{*}\right)$ in the wavelength range from 
700 to $2500 \mathrm{~nm}$ was calculated in accordance with the ASTM standard number E891-87. The NIR solar reflectance at wavelengths between 700 and $2500 \mathrm{~nm}$ that is reflected by a surface is the irradiance-weighted average of its spectral reflectance, $r(\lambda)$ in $\mathrm{W} \mathrm{m}^{-2}$ and can be determined from the eqn (1):

$$
R^{*}=\frac{\int_{700}^{2500} r(\lambda) i(\lambda) \mathrm{d}(\lambda)}{\int_{700}^{2500} i(\lambda) \mathrm{d}(\lambda)}
$$

where $r(\lambda)$ is obtained from the experiment and $i(\lambda)$ is the solar spectral irradiance $\left(\mathrm{W} \mathrm{m}^{-2} \mathrm{~nm}^{-1}\right)$ obtained from ASTM standard E891-87.

\subsection{Evaluation of antifungal activity}

The agar overlay method was used for the examination of the anti-fungal properties of APZO NHs/PMMA coated cotton fabrics. The effect of fungal growth was examined using the Aspergillus niger and Aspergillus flavus fungi species. In a typical experiment, the sterilized Petri-plate containing APZO NHs/PMMA coated cotton fabrics was overlaid with potato dextrose agar (Hi-media, Mumbai, India) containing the test fungal strains. The inhibitory effect was evaluated after incubation for $72 \mathrm{~h}$ at $37{ }^{\circ} \mathrm{C}$ and the results were evaluated by the measurement of inhibition zone around the APZO NHs/PMMA coated cotton fabrics.

\section{Results and discussion}

The main chemical constituent of a cotton fabric is cellulose, a macromolecular polymer, in which a long chain anhydro-betacellobiose building block is repeatedly present. The cellulose chains are mainly linked via hydrogen bonding. These hydrogen bonds occur between the hydroxyl groups of adjacent crystalline molecules of the cotton fiber. ${ }^{46}$ Hydroxyl groups in the cellobiose polymeric units are chemically reactive and organosilane molecules readily react with these hydroxyl groups. Silane coupling agents generally include alkoxy and alkyl bridges cross-linked through organofunctionalities. Hence, silane indeed plays a role as a chemical adhesive promoter for the fixation of nano $\mathrm{ZnO}$ with the cellulosic cotton surface through $\mathrm{NH}_{2}-\mathrm{OH}$ bonding. ${ }^{47}$ The organosilane is also a recognized surfactant/stabilizer to determine the growth of the $\mathrm{ZnO}$ particles that decide the $\mathrm{ZnO}$ colloidal stability. However, for the firm binding of nano $\mathrm{ZnO}$ with cotton fabrics, one has to have the optimum amount of organic silane capping and appropriate surface charge characteristics in addition to the controlled particle size.

The DLS particle size distribution and zeta potential analysis results observed for the silane modified $\mathrm{ZnO}$ hybrid particles along with their unmodified counterparts are illustrated in Fig. 2.

The average hydrodynamic diameter $\left(Z_{\text {ave }}\right)$ varies strongly with the surface-anchored amine functional groups present in organosilane. The $Z_{\text {ave }}$ values determined for the unmodified $\mathrm{ZnO}$ and silane treated hybrid $\mathrm{ZnO}$ are 337, 220, 239, and $385 \mathrm{~nm}$ for the respective silane concentrations of $0,1,2$, and $5 \mathrm{~mol} \%$. The corresponding size distribution curves are presented in the $\mathrm{ESI} \dagger$ (Fig. S1A). The size distribution is very narrow when the

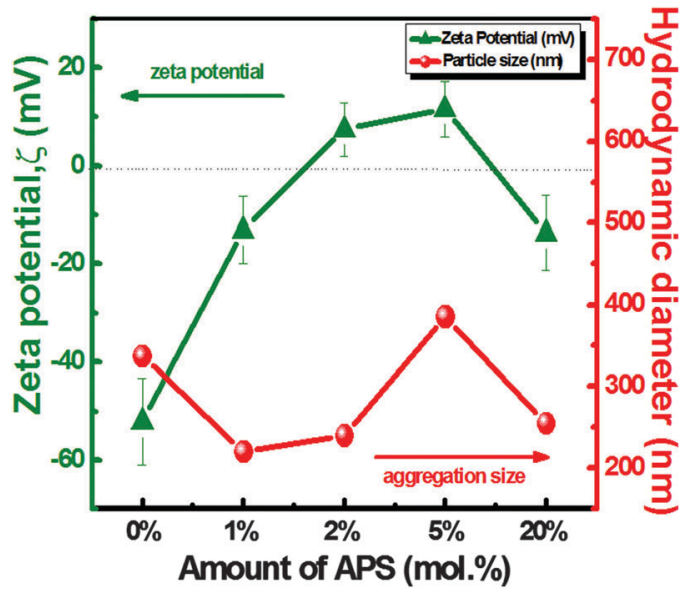

Fig. 2 Illustration of DLS particle size distribution and zeta potential measurements with respect to the different mol\% of APS in the APZO NHs.

$\mathrm{ZnO}$ nano particles are prepared by the in situ addition of silane. However, when an excess amount of silane was used, the particle size curve indicates a bi-modal size distribution. In this case, the particles fall in the range 255 and $970 \mathrm{~nm}$, respectively. The broad size distribution clearly indicated that in the absence of silane, the precipitation technique produced only $\mathrm{ZnO}$ nanoaggregates. Upon in situ silane addition, preferably with the least amount of silane, more specifically at $\mathrm{Si} / \mathrm{Zn}$ mol ratio $1 \%$, the size distribution is very narrow with an average particle size of $220 \mathrm{~nm}$. The size controlled growth of nano $\mathrm{ZnO}$ is attributed to the capping effect of the organosilane. Earlier studies on the synthesis of silane treated $\mathrm{ZnO}$ strongly confirm the capping effect of silane molecules to achieve size controlled growth of nano $\mathrm{ZnO}$ with tuned physicochemical properties. ${ }^{42}$ In one-pot synthesis, the strong chemical interaction of the organic ligands in APS controls the condensation reaction with $\mathrm{ZnO} / \mathrm{Zn}(\mathrm{OH})_{2}$ species. This further limits the ZnO self-condensation processes that ultimately decrease the particle growth. ${ }^{42}$ At higher loadings, the excess silane possibly produces a thick silanol capping layer due to the chemical cross-linking of the organo functional groups with the $\mathrm{ZnO}$ surface hydroxyls. This increases the tendency of localized aggregation finally leading to a bi-modal size distribution with a wide particle size range. The larger diameter in the DLS data can also be the effect of the outermost hydration layer of colloidal nanoparticles. ${ }^{48}$ The zeta-potential $(\zeta)$ analysis clearly describes the electrostatic interactions as well as the influence of surface charges in the single phase $\mathrm{ZnO}$ and silane treated $\mathrm{ZnO}$ hybrid colloidal particles. $\mathrm{ZnO}$ exhibits the isoelectric point (IEP) at pH values ranging between 8.7-10.3. ${ }^{49}$ Fig. 2 also shows the zeta potential trend in nano $\mathrm{ZnO}$ prepared with various amounts of silane molecules measured at $\mathrm{pH} 10$. Compared with unmodified nano $\mathrm{ZnO}$, the average zeta potential of the silane treated APZO NHs varied from -52.2 to -13.1 , 7.46, and $11.6 \mathrm{mV}$ with $0,1,2$, and $5 \mathrm{~mol} \%$ addition of APS respectively. The change of zeta potential suggests that the $\mathrm{ZnO}$ hybridization occurred with silanes depending on its concentration. The positively charged surface of APZO NHs is in fact essential for the chemical coupling of silane modified $\mathrm{ZnO}$ with the 

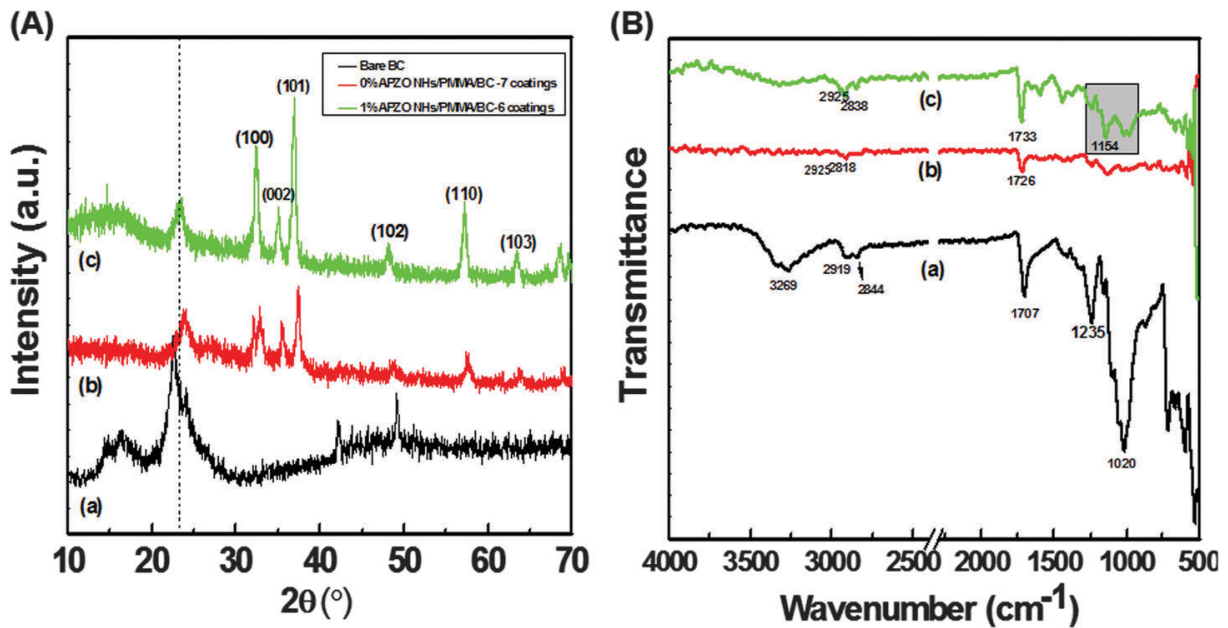

Fig. 3 (A) XRD pattern of (a) bare BC, (b) $0 \%$ APZO NHs/PMMA/BC-7 coatings, (c) 1\% APZO NHs/PMMA/BC- 6 coatings. (B) ATR-FTIR spectra of (a) bare BC, (b) $0 \%$ APZO NHs/PMMA/BC-7 coating, (c) $1 \%$ APZO NHs/PMMA/BC-7 coating.

highly hydrophilic linear cellulose micro fibrils. The hybridization of silane with ZnO is also clear from the FTIR analysis carried out on the APZO NHs given in the ESI $\dagger$ (Fig. S1B). The APZO NHs were further characterized and analyzed using SEM (Fig. S2), TEM (Fig. S3), TG (Fig. S4A), dispersion stability (Fig. S4B), UV-vis absorption spectra (Fig. S4C), PL spectra (Fig. S4D), and NIR reflectance spectra (Fig. S5) as shown in Tables S1 and S2 in the ESI. $\dagger$

The APZO NHs, when dispersed in a PMMA solution obtained by dissolving the polymer in a toluene medium, produced a stable organic-inorganic hybrid/polymer colloid. As expected the PMMA could not only physically fix the APZO NHs over the cotton surface, but also chemically interact with the cellulose through organosilane capping layers. Fig. 3A presents the XRD analysis of the cotton fabrics treated with APZO NHs/PMMA colloidal coatings. The bare cotton gives a broad crystalline peak at $2 \theta$ values between $15-16^{\circ}$ and a narrow peak at approximately $23^{\circ}$ representing the monoclinic cellulose unit cell with planes (101), (101), and (002) respectively, which matches with cellulose I structure (JCPDS no. 03-0226). ${ }^{50}$ When the cotton fabric is coated with APZO NHs/PMMA, the XRD shows six reflection peaks appearing at $2 \theta$ values of $31.9^{\circ}, 34.6^{\circ}, 36.5^{\circ}, 47.7^{\circ}, 56.8^{\circ}$, and $63.1^{\circ}$ corresponding to the planes (100), (002), (101), (102), (110), and (103), which could be indexed to the hexagonal wurtzite structure of $\mathrm{ZnO}$. The crystalline structure of nano $\mathrm{ZnO}$ is consistent with the standard JCPDS card no. 36-1451. The ATR-FTIR spectra in Fig. 3B further confirm the chemical interaction of APZO NHs/PMMA coatings with the cellulosic cotton fabrics. In uncoated cotton fabric, the characteristic bands corresponding to the hydroxyl contribution $(\mathrm{OH}$ stretching at $\left.\sim 3269 \mathrm{~cm}^{-1}\right)$ and C-H stretching $\left(\sim\right.$ at $\left.3000-2800 \mathrm{~cm}^{-1}\right)$ and the symmetric and asymmetric stretching mode of the cellulose functional group $\left(-\mathrm{CH}_{2}-\right)$ in the broad wavelength range $1000-1500 \mathrm{~cm}^{-1}$ are clearly observed. In silane modified ZnO embedded PMMA coated fabrics, the absorption bands at wavelengths 1154 and $1733 \mathrm{~cm}^{-1}$ clearly indicate the Si-O-Si and $\mathrm{C}=\mathrm{O}$ symmetrical stretching vibrations, strongly confirming the chemical hybridization of the APZO NHs/PMMA with the cotton surface. ${ }^{46}$

\subsection{Microstructures of APZO NHs/PMMA coatings}

The SEM images in Fig. 4 show the microstructures and the surface morphologies of the APZO NHs/PMMA coated and uncoated cotton fabrics. The SEM image corresponding to bare cotton is presented in Fig. 4A(a)-(c) at different magnifications. The image reveals a web-like network of cellulose fibrils. This woven network is composed of thick cellulose fibril bundles. Each fiber bundle has a thickness of about 50 microns in which an individual fibril has a width of $5 \mu \mathrm{m}$. The fiber texture, its physical length and the orientation are clear from the magnified images (Fig. 4A(b) and (c)). Interwoven cotton fabrics have micrometer size voids between the fiber bundles. The fibrous structural framework is also micro-porous. The penetration of light energy could be easily realized by this porous material. The air trapped in the micro-capillaries of cellulose fibers absorbs heat energy when they are exposed to strong solar heat and builds the surface temperature over cotton textiles. A mildly applied homogenous coating prepared out of any active nanoscale functional material can completely cover the porous cellulose fabrics and probably permit only the light to transfer but effectively avoid the energy absorption depending upon its optical properties. The SEM images in Fig. $4 \mathrm{~B}(\mathrm{a})-(\mathrm{c})$ represent the black cotton the with neat PMMA solution applied. It can be seen that the PMMA adhesion with the cellulosic fabric is firmly strong. The methylene bonded carbon functional group in the PMMA polymer aids bridging with the hydroxyl group and chemically adheres on the cotton fabric. At higher magnifications, the neat PMMA coatings appear porous. The pore size is micrometer size. The evaporation of solvent molecules resulted in nearly homogeneous porous coatings. However, the SEM image explicitly shows the PMMA film adhered on cellulose surface. The porous nature is seen only above these continuously covered films. Fig. $4 \mathrm{C}(\mathrm{a})-(\mathrm{c})$ represents the microstructures of the $\mathrm{ZnO} / \mathrm{PMMA}$ coatings without any silane. In the absence of silane, the coatings 
(A)

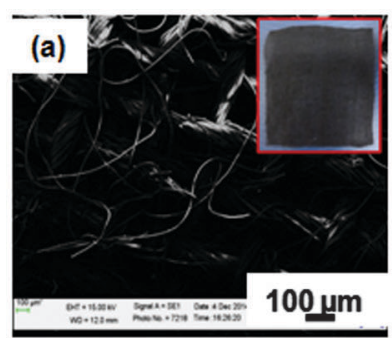

(B)

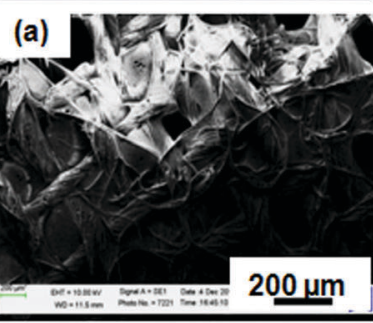

(C)

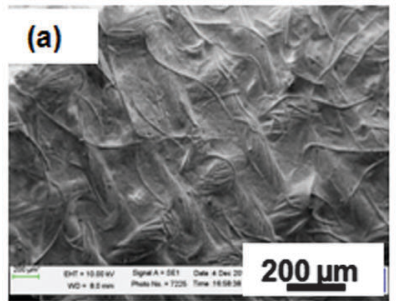

(D)

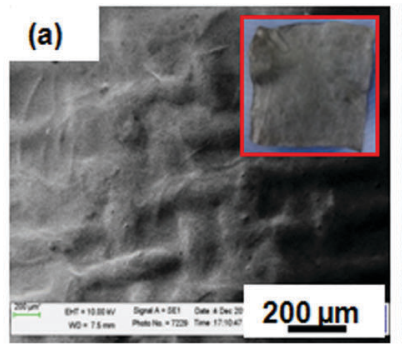

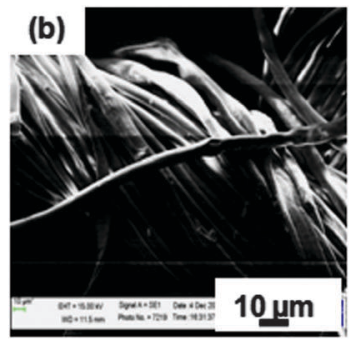
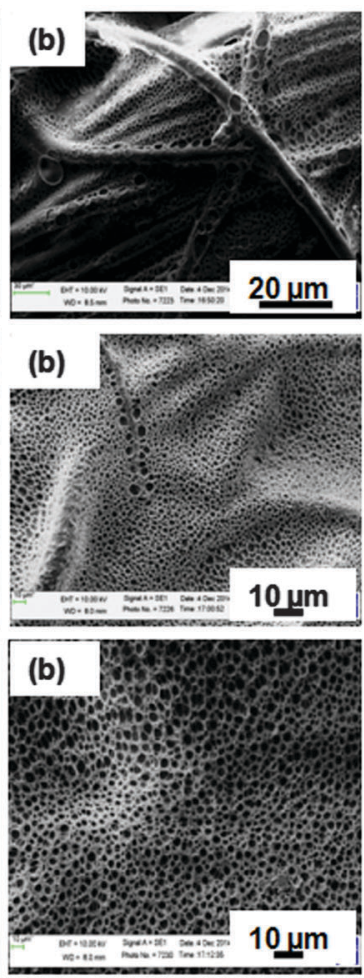
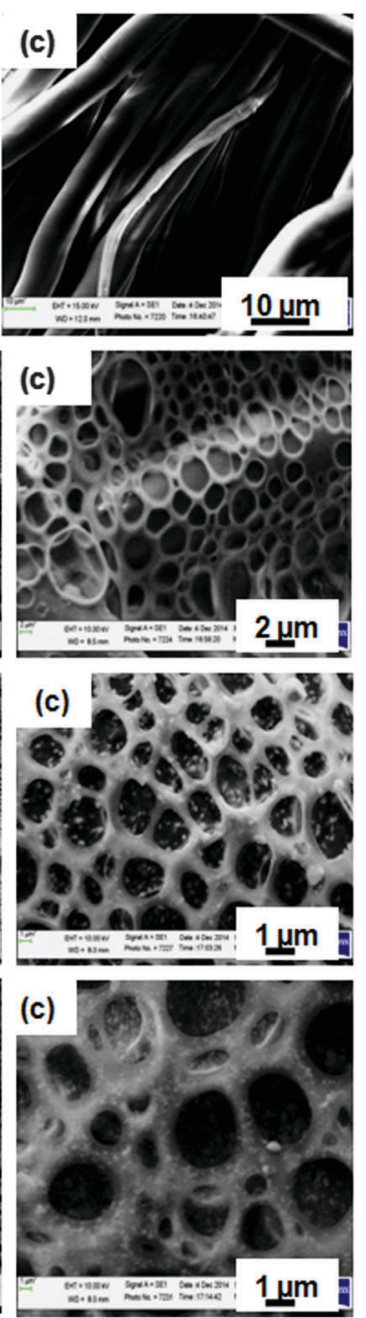
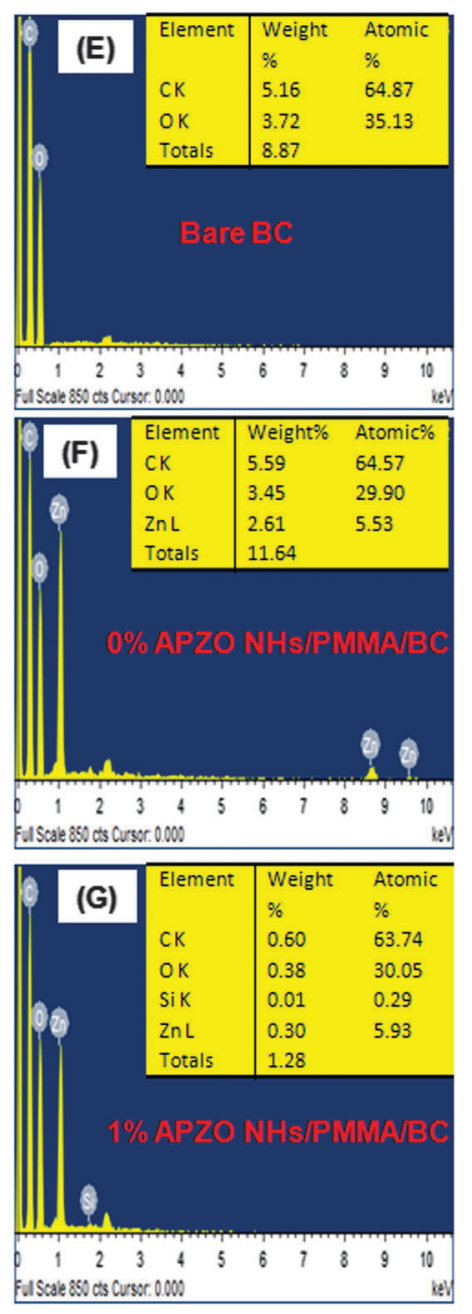

Fig. 4 SEM images shown: (A) bare BC, (B) PMMA/BC, (C) 0\% APZO NHs/PMMA/BC-6 coating and (D) 1\% APZO NHs/PMMA/BC-6 coating. The different magnifications of the corresponding samples are also shown in (a-C). The EDX patterns show the elemental analysis of (E) bare BC, (F) 0\% APZO NHs/ PMMA/BC-6 coating, and (G) $1 \%$ APZO NHs/PMMA/BC-6 coating.

have a rough surface texture. When the coatings are made with the silane modified $\mathrm{ZnO} \mathrm{NHs} / \mathrm{PMMA}$ coatings (Fig. $4 \mathrm{D}(\mathrm{a})-(\mathrm{c})$ ), they become physically smooth and in these smooth coatings the hybrid particulates are deeply penetrated into the inter-fiber voids and dispersed along the continuous layers. The coated layers have highly homogeneous coverage of the cotton fabrics. In both cases, the magnified images apparently show the firm sticking of the nanosize $\mathrm{ZnO}$ particles, although some of them are micro-aggregates, on to the textile fibrils. One notable feature observed from these SEM images is the hierarchical porosity of the multilayered coatings. One can have the following advantages in this kind of nanohybrid embedded PMMA coatings. First of all, the porous feature of the coatings effectively allows the nano $\mathrm{ZnO}$ to directly interact with the incident light. The PMMA polymer topcoat makes the fabric hydrophobic in nature. Its porous multilayer architecture resembles the membrane like surface that allows the light and promotes the convection air-flow for passive cooling. In this work, it is seen that the silane treated $\mathrm{ZnO}$ nanohybrids encapsulated in the PMMA matrix which is embedded in the cotton fabrics finally resulted in hydrophobic, photoactive topcoats. The chemical composition of the coated surface of the fabrics was analyzed by EDX spectroscopy. It confirmed that the hybrid coatings have elemental $\mathrm{Zn}, \mathrm{O}$ and Si atoms. The SEM microstructures strongly support the effective impregnation of the APZO NHs/PMMA colloidal sol through the micro capillaries of the cellulosic cotton fibers. Morphological and elemental analysis of the white cotton fabrics 0\% APZO NHs/PMMA/WC and 1\% APZO NHs/PMMA/WC are shown in the ESI $\dagger$ (Fig. S6).

An insight into the surface smoothness of the APZO NHs/PMMA coatings on cotton fabrics can be seen from the AFM images presented in Fig. 5. In 1 mol\% silane modified ZnO/PMMA coatings, the pore depth seems to decrease roughly $50 \%$. The pore depth was about $2.7 \mu \mathrm{m}$ in $0 \%$ silane treated $\mathrm{ZnO} / \mathrm{PMMA}$ coatings. However, the pore depth was decreased to $1.4 \mu \mathrm{m}$ in the presence of silane treated coatings. The controlled rate of evaporation in chemically bridged APZO NHs/PMMA colloids is a plausible reason for the evolution of fine pores with restricted 
(A)

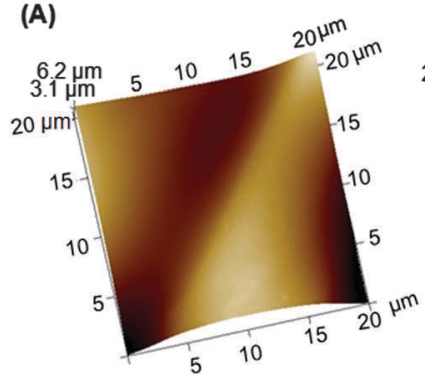

(B)

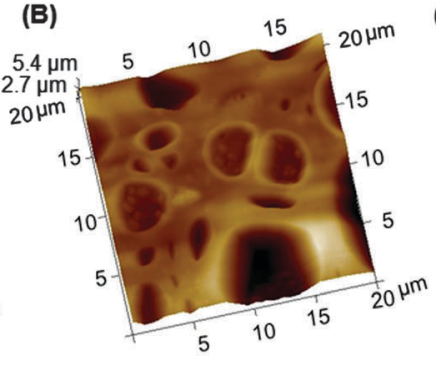

(C)

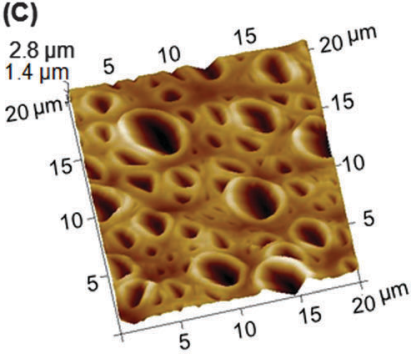

Fig. 5 3D AFM topographic images of (A) bare black cotton, (B) 0\% APZO NHs/PMMA/BC-3 coating, and (C) 1\% APZO NHs/PMMA/BC-3 coating.

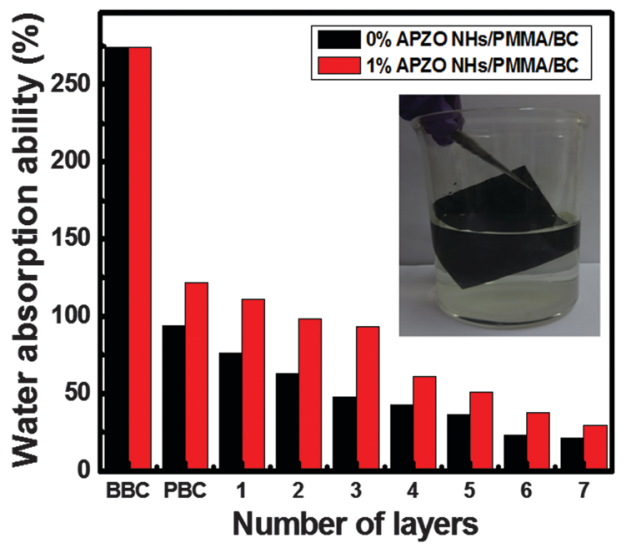

Fig. 6 Water absorption ability of $0 \%$ and $1 \%$ APZO NHs/PMMA/BC samples with respect to the number of layers. (BBC = bare black cotton, $\mathrm{PBC}=$ neat PMMA coated cotton). Inset shows the photographic image of black cotton fabrics immersed in water.

overall pore width and pore wall thickness that contribute the decreased roughness.

The water absorption property of untreated cotton and coatings made with APZO NHs/PMMA sol was tested against simple $\mathrm{ZnO} /$ PMMA and the results are compared in Fig. 6 . The percentage water absorption was calculated according to the following equation; $w \%=w_{\mathrm{a}}-w_{\mathrm{o}} / w_{\mathrm{o}} \times 100$, where $w \%$ is the water absorption ability of the fabrics, $w_{\mathrm{a}}$ is the mass of the fabric with absorbed water, and $w_{\mathrm{o}}$ is the mass of the dried fabric samples. This test demonstrates that the layers prepared with hybrid nanoparticles have comparatively better hydrophobicity. The degree of the hydrophobic nature indirectly indicates the homogeneous coverage of the hybrid films at the interface over cellulose capillaries and intervoid-cavities.

\subsection{UV shielding effect of black cotton fabrics with APZO NHs/PMMA coatings}

The UV-vis absorbance spectra of the APZO NHs/PMMA coated on cotton fabrics, via LbL technique, were measured in the wavelength region $200-800 \mathrm{~nm}$ using the bare cotton fabrics as reference. The UV absorption analysis shows that the bare cotton and the neat PMMA coatings are highly transparent to UV rays. The cotton textiles treated with ZnO and dispersed PMMA and with silane modified nano ZnO hybrid embedded PMMA coatings exhibit absorption of UV radiation at the wavelengths 364 and $349 \mathrm{~nm}$, respectively as evidenced from the UV absorption spectra provided in Fig. 7A and B. Greater UV absorption is seen with 5 layers of hybrid coatings on to the cotton fabrics. UV absorption by the multilayer hybrid coatings takes place at the same wavelength as that of the dispersion of nano $\mathrm{ZnO}$ indicating that the hybrid nanoparticles preserve their molecular state even
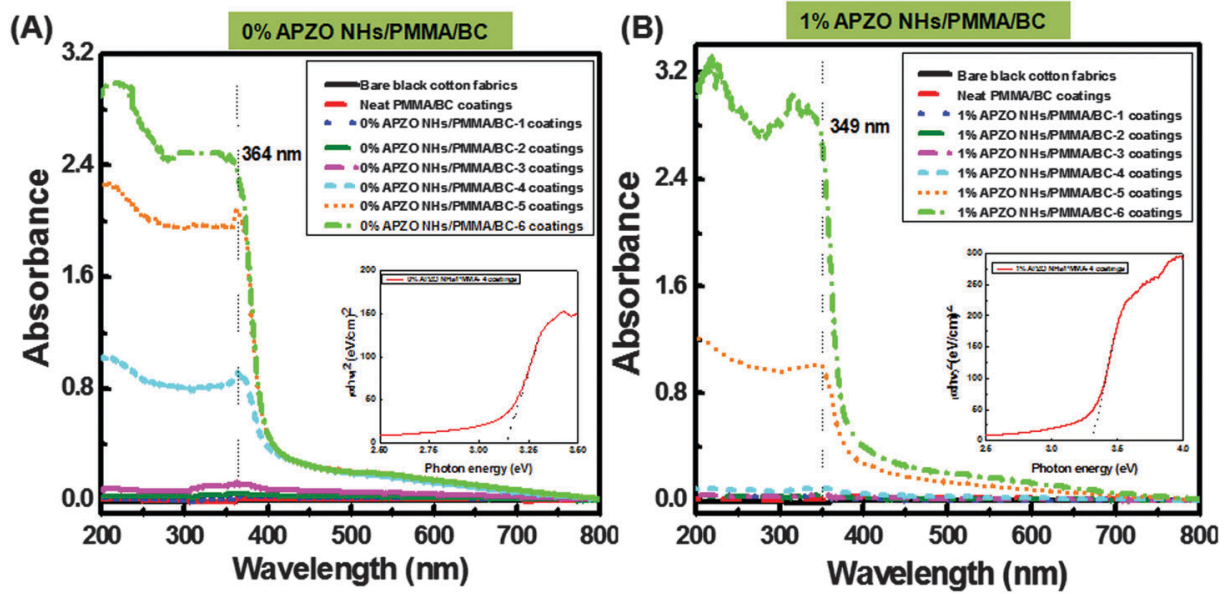

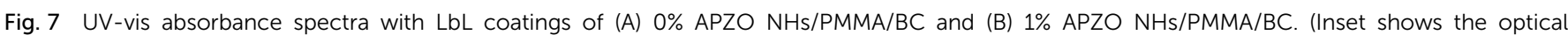
absorption spectra for photon energy in terms of eV.) 
after they are modified with silane organics and embedded in the PMMA polymer matrix. The effective capping of APS is evidenced by the blue-shift of the wavelength at $349 \mathrm{~nm}$. The inset in Fig. 7 illustrates the bandgaps of the $0 \%$ APZO NHs/PMMA/BC and 1\% APZO NHs/PMMA/BC coatings which were computed as 3.13 and $3.32 \mathrm{eV}$, respectively. Apparently, it can be understood that the nano $\mathrm{ZnO}$ and APZO NH embedded PMMA coatings on cotton fabrics can efficiently block the UV and produce safe textiles.

\subsection{NIR reflectance and total solar reflectance $\left(R^{*}\right)$ of coated cotton fabrics}

NIR reflectance of black as well as white colored cotton fabrics treated with the APZO NHs/PMMA sol coatings are shown in Fig. 8 and 9. Overall, the bare as well as the light transparent PMMA coated black color cotton surface shows less than $30 \%$ NIR reflectance. At the same time, when compared to the black color cotton fabrics, the white cotton has about 50\% NIR reflectance by virtue of its bright surface, though the fabric texture is identical. In both cotton fabrics, the level of diffuse NIR reflectance is decreased with increasing wavelength in the region 1500-2500 $\mathrm{nm}$. When nano $\mathrm{ZnO}$ and silane modified nano ZnO hybrid embedded PMMA coatings are applied over the black fabric, the reflectance is increased from the low value of $30 \%$ to high value of $50 \%$, close to that of white cotton fabrics, without affecting its black appearance. It is seen that the coating layers strongly enhance NIR reflectance. Greater numbers of APZO NHs/PMMA coatings lead to better reflectance because of the increased concentration of $\mathrm{ZnO}$ nanoparticles embedded in the multilayer coatings. The 1\% APZO NHs/PMMA/BC-3 layers coatings show $\sim 53$ and 50\% NIR reflectance at high heat energy carrying IR wavelengths 810 and $1100 \mathrm{~nm}$, respectively (Fig. 9C). The same samples show NIR solar reflectance, $R^{*}$, as 48,49 , and $52 \%$ for 3, 5, and 7 layers coatings respectively (Fig. 9D). The weak Rayleigh scattering or strong Mie scattering determines the optical properties of a material when solar light energy interacts with the material, depending upon its crystalline arrangement. In cotton fabrics, the interaction with the light energy is weak due to its visible opaqueness while strong Mie scattering can be possible. If the material contains any nanoscale optically transparent elements, depending upon the nano-dimensions, the light can be absorbed or transmitted. Cotton fabric is composed of parallelaligned micro fibrils and SEM images indicate the cellulosic fibrediameter has a size of less than one micron. This micrometer range of the microfibrils is in fact inadequate for the effective reflection of the near IR waves in the critical region 800-1100 nm.
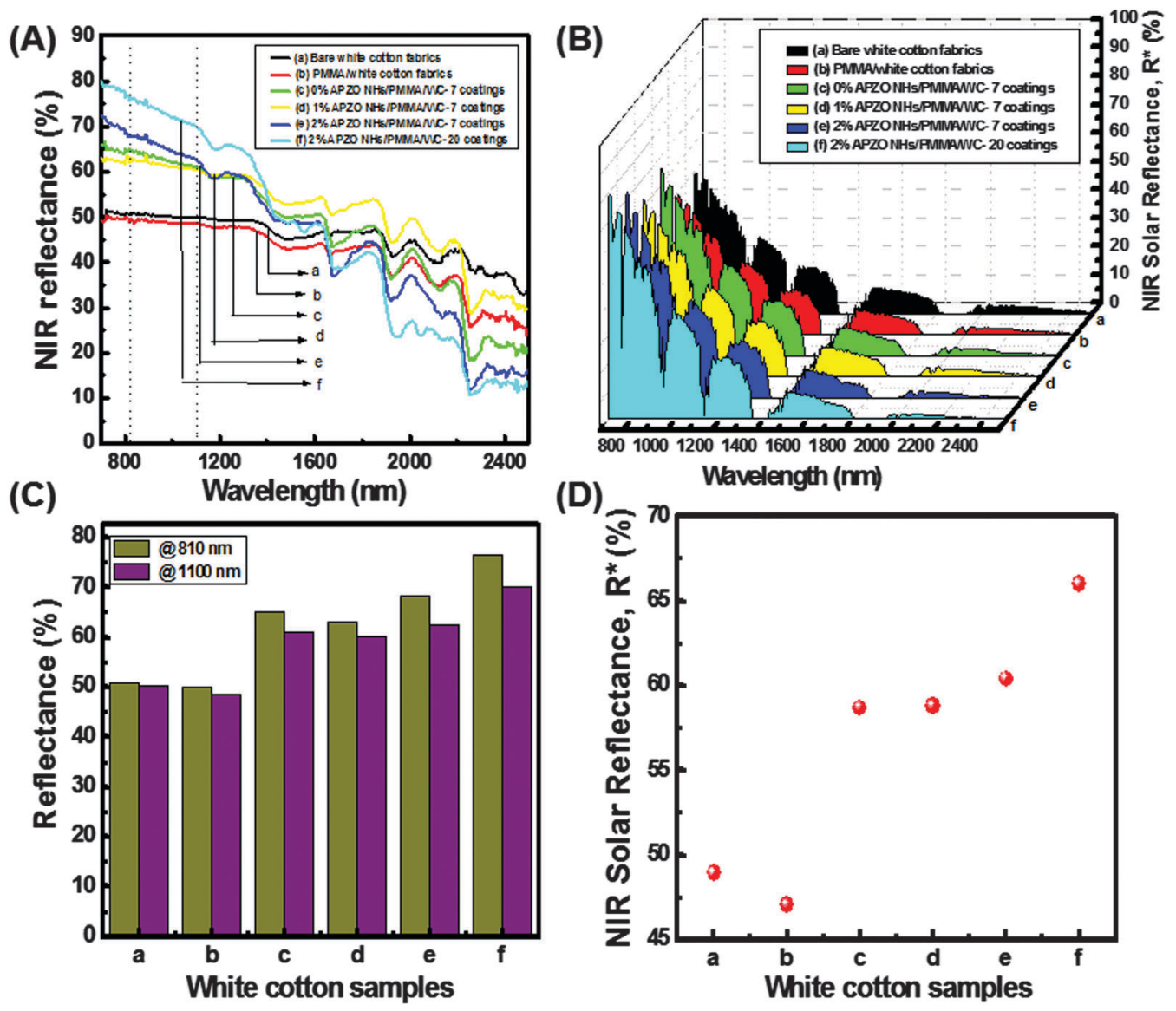

Fig. 8 (A) NIR reflectance of APZO NHs/PMMA treated white cotton fabrics, (B) NIR solar reflectance of corresponding samples. (C) Reflectance at 810 and $1100 \mathrm{~nm}$. (D) NIR solar reflectance vs. white cotton samples. (a) Bare white cotton fabrics, (b) PMMA/WC, (c) $0 \%$ APZO NHs/PMMA/WC - 7 coatings, (d) $1 \%$ APZO NHs/PMMA/WC - 7 coatings, (e) $2 \%$ APZO NHs/PMMA/WC -7 coatings, (f) $2 \%$ APZO NHs/PMMA/WC - 20 coatings. 

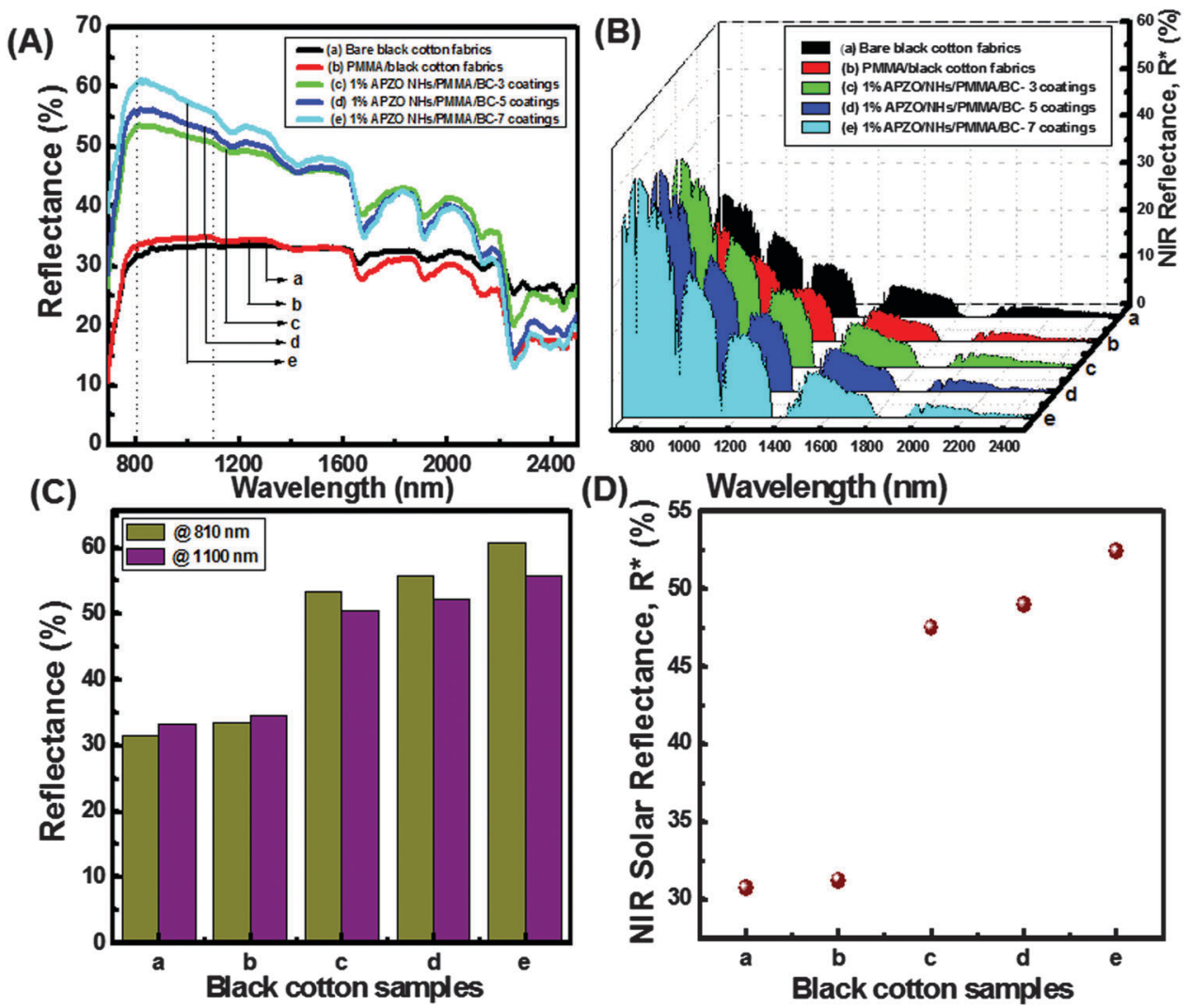

Fig. 9 (A) NIR reflectance of APZO NHs/PMMA coated black cotton fabrics, (B) NIR solar reflectance of corresponding samples. (C) Diffuse reflectance (a 810 and $1100 \mathrm{~nm}$. (D) NIR solar reflectance vs. black cotton samples. (a) Bare BC, (b) PMMA/BC, (c) 1\% APZO NHs/PMMA/BC-3 coatings, (d) $1 \%$ APZO $\mathrm{NHs} / \mathrm{PMMA} / \mathrm{BC}-5$ coatings, (e) $1 \%$ APZO NHs/PMMA/BC-7 coatings.
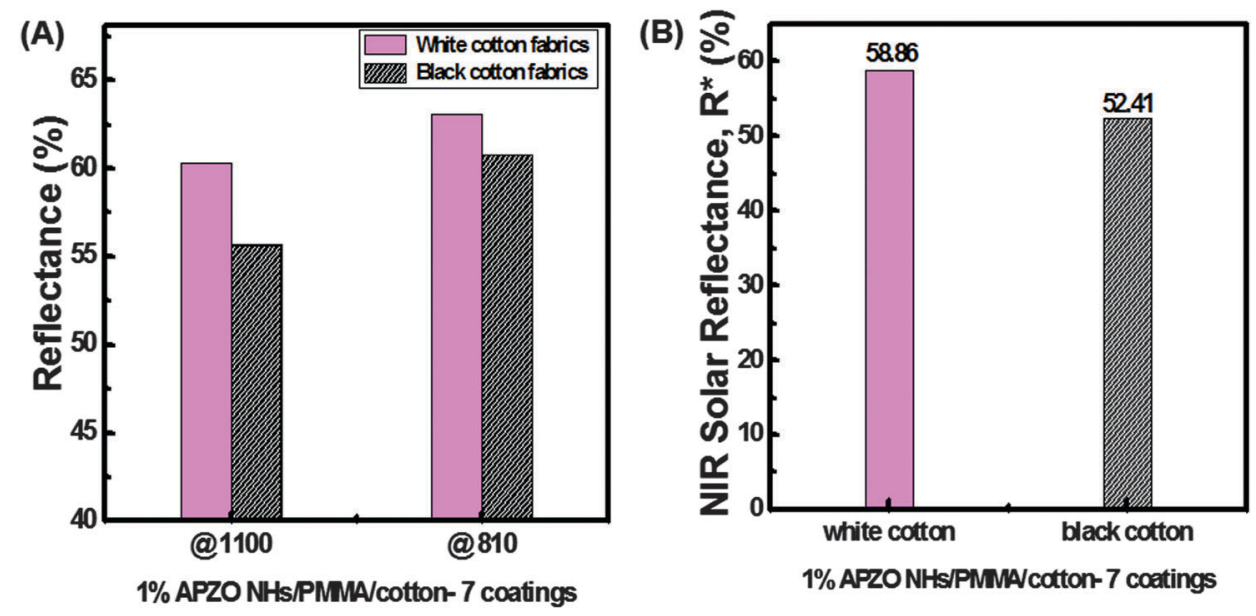

(C)
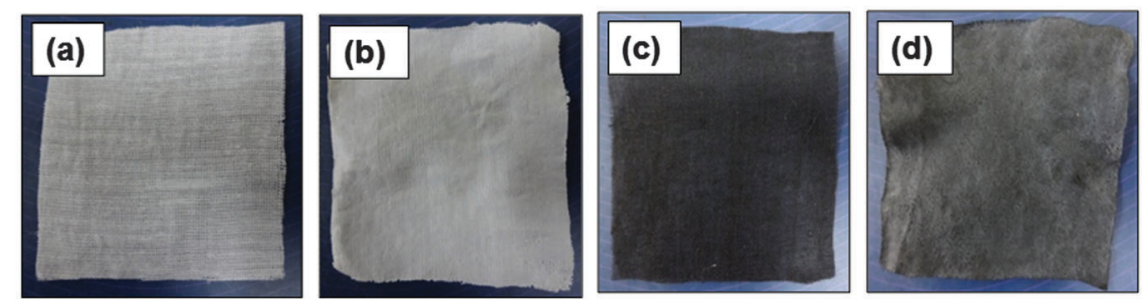

Fig. 10 (A) Near-IR reflectance of 1\% APZO NHs/PMMA coated black and also white cotton fabrics at 810 and $1100 \mathrm{~nm}$. (B) NIR solar reflectance of black and also white cotton fabrics treated with $1 \%$ APZO NHs/PMMA-7 coatings. (C) Photographic images of cotton fabrics: (a) bare WC, (b) $1 \%$ APZO $\mathrm{NHs} / \mathrm{PMMA} / \mathrm{WC}-7$ coatings, (c) bare BC and (d) $1 \% \mathrm{APZO}$ NHs/PMMA/BC -7 coatings. 
Moreover, another important criteria for the light-material interaction is the matching refractive index values of the material. ${ }^{1}$ In the cotton fabric crystalline domains, the cellulose chains are connected by hydrogen bonds to form a highly ordered crystalline structure. The refractive index of cellulose is 1.618 along the fiber and 1.544 in the transverse direction. This value was enhanced when an APZO NHs embedded PMMA topcoat was provided. It is a nanocomposite coating and the high refractive index of active nano $\mathrm{ZnO}$ contributes to high reflection of near-IR energy. The high refractive index and loosely agglomerated nanoaggregates with a particle diameter in the range 250-350 $\mathrm{nm}$ have a combined effect on the NIR reflectance of the APZO NHs/PMMA coatings.

Fig. 10 depicts the comparative NIR reflectance and NIR solar reflectance performances of the white cotton fabrics in comparison with their black color counterpart prepared with $1 \%$ APZO NHs/PMMA coatings having 7 layers. The white cotton fabrics show 63 and 60\% NIR reflectance. The corresponding values for black cotton fabrics are 60 and 55\% at wavelengths of 810 and $1100 \mathrm{~nm}$, respectively (Fig. 10A). The NIR solar reflectance $\left(R^{*}\right)$ of white as well as black fabrics modified with $1 \%$ APZO NHs/PMMA/cotton-7 coatings were evaluated as 58 and $52 \%$, respectively. It can be concluded that irrespective of the surface of the cotton textiles, the APZO NH embedded PMMA coatings can enhance the NIR solar reflectance (Fig. 10B). Photographic images of the uncoated and coated white and black cotton fabrics are also shown in Fig. 10C. A photograph of the cotton textile coated with 7 layers of 1\% APZO NHs/PMMA/BC showing good flexibility is shown in the ESI, $\dagger$ Fig. S7.

\subsection{Evaluation of antifungal activity}

Fig. 11 shows the antifungal property of the cotton textiles coated with the APZO NHs/PMMA colloid materials. Compared to the bare cotton fabrics, the APZO NHs dispersed PMMA matrix coated cotton textiles distinctly exhibit better zones of inhibition of the test fungi species Aspergillus niger and Aspergillus flavus. Cotton fabrics are microporous and their surfaces are hydrophilic. In any humid environment, the absorption of moisture favors the growth of fungi. Fungus is in general one of the factors responsible for allergies, asthma and cough. The silane modified $\mathrm{ZnO}$ hybrid embedded PMMA coatings not only introduce hydrophobicity in cotton fabrics for effective moisture repellence qualities but also offer resistance against fungal growth, indicating that the APZO NHs/PMMA modified cotton textiles perform as bio-safe cool textiles.

Plausible antimicrobial mechanism. Previous studies on the antibacterial behavior of zinc oxide NPs confirmed that their

\section{Aspergillus niger}

(A)
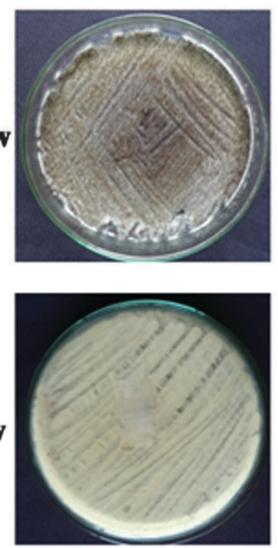

Back view

\section{Aspergillus flavus}
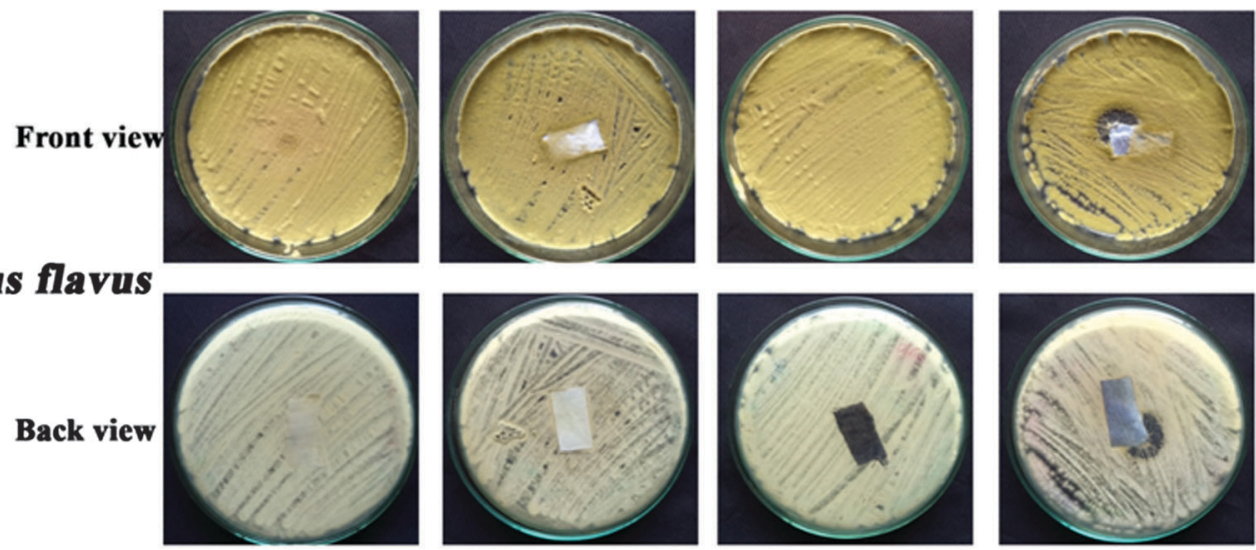

Fig. 11 Antifungal activities of (A) bare white cotton fabrics, (B) 1\% APZO NHs/PMMA coated white cotton fabrics, (C) bare black cotton fabrics, (D) $1 \%$ APZO NHs/PMMA coated black cotton fabrics. 
morphology and oxidative stress play important roles in the antibacterial activity. ${ }^{51,52}$ Due to the semiconducting nature of ZnO (band gap $3.2 \mathrm{eV}$ ), it acts as photocatalytic material. Superoxide anion production due to photocatalytic reactions is one of the probable reasons for disturbing microbial processes, which kills microorganisms including bacteria and fungi. With the combined effects of the hydrophobic nature and photo-active properties of $\mathrm{ZnO}$, the hybrid coatings showed excellent antifungal properties. ${ }^{53}$

\section{Conclusions}

In summary, a novel chemically reactive, multifunctional hybrid/ polymer nanocoatings were designed with silane treated $\mathrm{ZnO} /$ PMMA which is validated on black colored cotton fabrics. The nanohybrid/polymer topcoats offer beneficial properties such as UV absorption, enhanced NIR reflectance, hydrophobicity and antifungal properties. Co-precipitation synthesis of $\mathrm{ZnO}$ in the presence of in situ organosilane resulted in a nano $\mathrm{ZnO}$ hybrid with particle size in the range $220-350 \mathrm{~nm}$. The formation of silanol functional groups on the surface of the nano $\mathrm{ZnO}$ produced positively charged surfaces that chemically react with the hydroxyl groups present in the cellulosic building blocks which promotes firm physico-chemical adhesion of nano $\mathrm{ZnO}$ over the cellulosic fibrils through chemical cross-linking. A multilayer porous hybrid coating consisting of APZO NHs/PMMA colloids and the hybrid sol is dip-coated on cotton surface. The hybrid topcoats enhanced the NIR reflectance of the bare black cotton fabric from 30 to $63 \%$ when the coating layer was applied 7 times and had inherent flexibility. The hybrid coating also makes the textile less prone to water absorption and more resistant against fungal growth. Upon coating with silane treated $\mathrm{ZnO}$ embedded PMMA hybrids, the black cotton fabric finally attained NIR reflectance close to that of the white cotton textiles.

\section{Acknowledgements}

The authors thank the Director, CSIR-NIIST for providing laboratory facilities. One of the authors S. Soumya is grateful to University Grant Commission (UGC), Government of India, New Delhi for awarding Senior Research Fellowship. The authors acknowledge Dr M. L. P. Reddy, Ms Lucy Paul, Mr Aswin, and Mr Kiran Mohan for their help with material characterizations.

\section{References}

1 P. Jeevanandam, R. S. Mulukutla, M. Phillips, S. Chaudhuri, L. E. Erickson and K. J. Klabunde, J. Phys. Chem. C, 2007, 111, 1912-1918.

2 R.-D. Wendel, US Pat. 8932965 B1, 2015.

3 A. Wong, A.-W. Daoud, H. Liang and Y. S. Szeto, Sol. Energy Mater. Sol. Cells, 2015, 134, 425-437.

4 R. Y. Jang, US Pat. 0154482 A1, 2014.

5 D. Miao, A. Li, S. Jiang and S. Shang, Ceram. Int., 2015, 41, 6312-6317.
6 N. Abidi, L. Cabrales and E. Hequet, ACS Appl. Mater. Interfaces, 2009, 10, 2141-2146.

7 M. Yu, Z. Wang, H. Liu, S. Xie, J. Wu, H. Jiang, J. Zhang, L. Li and J. Li, ACS Appl. Mater. Interfaces, 2013, 5, 3697-3703.

8 R. H. Wang, X. W. Wang and J. H. Xin, ACS Appl. Mater. Interfaces, 2010, 1, 82-85.

9 Y. Li, J. Liu, J. Liang, X. Yu and D. Li, ACS Appl. Mater. Interfaces, 2015, 7, 6574-6583.

10 R. H. Wang, J. H. Xin and X. M. Tao, Inorg. Chem., 2005, 44, 3926-3930.

11 M. K. Mehrizi, S. M. Mortazavi, S. Mallakpour and S. M. Bidoki, Color Res. Appl., 2012, 37, 199-205.

12 W. Duan, A. Xie, Y. Shen, X. Wang, F. Wang, Y. Zhang and J. Li, Ind. Eng. Chem. Res., 2011, 50, 4441-4445.

13 J. Manna, S. Goswami, N. Shilpa, N. Sahu and R. K. Rana, ACS Appl. Mater. Interfaces, 2015, 7, 8076-8082.

14 X. Liu, L. Ge, W. Li, X. Wang and F. Li, ACS Appl. Mater. Interfaces, 2015, 7, 791-800.

15 D. Xiong and G. Liu, Langmuir, 2012, 28, 6911-6918.

16 J. Johnson, J. Heidenreich, R. Mantz, P. Baker and M. Donley, Prog. Org. Coat., 2003, 47, 432-442.

17 Y.-Q. Li, S.-G. Mei, Y.-J. Byon, J.-L. Wang and G.-L. Zhang, ACS Sustainable Chem. Eng., 2014, 2, 318-321.

18 S. Balanand, M. J. Maria, T. P. D. Rajan, A. P Mohamed and S. Ananthakumar, Chem. Eng. J., 2016, 284, 657-667.

19 R. Levinson, P. Berdahl, H. Akbari, W. Miller, I. Joedicke, J. Reilly, Y. Suzuki and M. Vondran, Sol. Energy Mater. Sol. Cells, 2007, 91, 304-314.

20 R. Levinson, H. Akbari, P. Berdahl, K. Wood, W. Skilton and J. Petersheim, Sol. Energy Mater. Sol. Cells, 2010, 94, 946-954.

21 P. Petkova, A. Francesko, M. M. Fernandes, E. Mendoza, I. Perelshtein, A. Gedanken and T. Tzanov, ACS Appl. Mater. Interfaces, 2014, 6, 1164-1172.

22 I. Perelshtein, G. Applerot, N. Perkas, E. Wehrschetz-Sigl, A. Hasmann, G. M. Guebitz and A. Gedanken, ACS Appl. Mater. Interfaces, 2009, 2, 361-366.

23 M. L. Shiao, K. C. Hong, H. M. Kalkanoglu and G. F. Jacobs, US Pat. 8361597 B2, 2013.

24 E. Viasnoff and M. L. Shiao, US Pat. 8277943 B2, 2012.

25 E. Viasnoff, US Pat. 8623499 B2, 2014.

26 H. Hwang, J. H. Park and B. G. Kim, US Pat. 0059148 A1, 2013.

27 J. Shi, H. L. Karlsson, K. Johansson, V. Gogvadze, L. Xiao, J. Li, T. Burks, A. Garcia-Bennett, A. Uheida, M. Muhammed, S. Mathur, R. Morgenstern, V. E. Kagan and B. Fadeel, ACS Nano, 2012, 6, 1925-1938.

28 Y. Gao, I. Gereige, A. E. Labban, D. Cha, T. T. Isimjan and P. M. Beaujuge, ACS Appl. Mater. Interfaces, 2014, 6, 2219-2223.

29 L. Wu, J. Zhang, B. Lia and A. Wanga, J. Mater. Chem. B, 2013, 1, 4756-4763.

30 X. Wang, S. Zhou and L. Wu, J. Mater. Chem. C, 2013, 1, 7547. 31 S. Soumya, P. A. Mohamed, K. Mohan and S. Ananthakumar, Sol. Energy Mater. Sol. Cells, 2015, 143, 335-346.

32 S. Soumya, P. A. Mohamed, L. Paul, K. Mohan and S. Ananthakumar, Sol. Energy Mater. Sol. Cells, 2014, 125, 102-112. 
33 N. Kiomarsipour, R. S. Razavi, K. Ghani and M. Kioumarsipour, Appl. Surf. Sci., 2013, 270, 33-38.

34 Y. Zhang, X. Wang, Y. Liu, S. Song and D. Liu, J. Mater. Chem., 2012, 22, 11971.

35 K. Matsuyama, K. Mishima, T. Kato, K. Irie and K. Mishima, J. Colloid Interface Sci., 2012, 367, 171-177.

36 S. Li, M. S. Toprak, Y. S. Jo, J. Dobson, D. K. Kim and M. Muhammed, Adv. Mater., 2007, 19, 4347-4352.

37 M. Eita, L. Wagberg and M. Muhammed, ACS Appl. Mater. Interfaces, 2012, 4, 2920-2925.

38 C. Jiang, M. Cheng, H. Liu, L. Shao, X. Zeng, Y. Zhang and F. Shi, Ind. Eng. Chem. Res., 2013, 52, 13393-13400.

39 S. Chen, X. Li, Y. Li and J. Sun, ACS Nano, 2015, 9, 4070-4076.

40 F. Carosio, G. Fontaine, J. Alongi and S. Bourbigot, ACS Appl. Mater. Interfaces, 2015, 7, 12158-12167.

41 M. Busila, V. Musat, T. Textor and B. Mahltig, RSC Adv., 2015, 5, 21562-21571.

42 D. Costenaro, F. Carniato, G. Gatti, L. Marchese and C. Bisio, New J. Chem., 2013, 37, 2103-2109.
43 N. Serpone, D. Lawless and R. Khairutdinov, J. Phys. Chem., 1995, 99, 16646-16654.

44 S. Jose, A. Prakash, S. Laha, S. Natarajan and M. L. P. Reddy, Dyes Pigm., 2014, 107, 118-126.

45 S. Sangeetha, R. Basha, K. J. Sreeram, S. N. Sangilimuthu and B. U. Nair, Dyes Pigm., 2012, 94, 548-552.

46 C. Chung, M. Lee and E. K. Choe, Carbohydr. Polym., 2004, 58, 417-420.

47 Y. Xie, C. A. S. Hill, Z. Xiao, H. Militz and C. Mai, Composites, Part A, 2010, 41, 806-819.

48 J. Liu, X. Yang, K. Wang, X. He, Q. Wang, J. Huang and Y. Liu, ACS Nano, 2012, 6, 4973-4983.

49 R. Marsalek, APCBEE Proc., 2014, 9, 13-17.

50 T. J. Athauda, P. Hari and R. R. Ozer, ACS Appl. Mater. Interfaces, 2013, 5, 6237-6246.

51 D. Sourabh, W. Rizwan, K. Farheen, K. M. Yogendra, M. Javed and A. A. Abdulaziz, PLoS One, 2014, 9, 111289.

52 R. R Krishna, T. K. Ranjit and C. M. Adhar, Langmuir, 2011, 27, 4020-4028.

53 G. R. Navale, M. Thripuranthaka, D. J. Late and S. S. Shinde, JSM Nanotechnol. Nanomed., 2015, 3(1), 1033. 